\title{
Electronic ordering and the management of treatment interdependencies: a qualitative study of paediatric chemotherapy
}

\author{
Valentina Lichtner ${ }^{1,2^{*}}$ D, Bryony Dean Franklin ${ }^{2,3}$, Luciano Dalla-Pozza ${ }^{4}$ and Johanna I. Westbrook ${ }^{1}$
}

\begin{abstract}
Background: There are serious safety risks associated with chemotherapy, often associated with interdependencies in regimens administered over months or years. Various strategies are used to manage these risks. Computerized provider order entry (CPOE) systems are also implemented to improve medication safety. Little is known regarding the effect of CPOE on how clinicians manage chemotherapy interdependencies and their associated safety strategies.

Methods: We conducted a multi-method qualitative study in a paediatric hospital. We analysed 827 oncology incidents reported following CPOE implementation and carried out semi-structured interviews with doctors $(n=10)$, nurses $(n=6)$, a pharmacist, and oncology CPOE team members $(n=2)$. Results were interpreted according to safety models (ultra-safe, high-reliability organisations [HROs], or ultra-adaptive).

Results: Incident reports highlighted two interrelated types of interdependencies: those within organisation of clinical activities and those inherent in chemotherapy regimens. Clinicians reported strategies to address chemotherapy risks and interdependencies. These included rigid rules and 'no go' contexts for treatment to proceed, typical of the ultra-safe model; use of time (e.g. planning only so far ahead) and sensitivity to operations, typical of HROs. We identified three different time horizons in CPOE use in relation to patients' treatments: life-long, the whole regimen, and the 'here and now'. CPOE supported ultra-safe strategies through automation and access to rules/standardisation, but also created difficulties and contributed to incidents. It supported the 'here and now' better than a life-long or whole regimen view of a patient treatment. Sensitivity to operations was essential to anticipate and resolve uncertainties, hazards, CPOE limitations, and mismatches between CPOE processes and workflow in practice.

(Continued on next page)
\end{abstract}

\footnotetext{
* Correspondence: v.lichtner@ucl.ac.uk; valentina.lichtner@mq.edu.au

${ }^{1}$ Australian Institute of Health Innovation, Faculty of Medicine, Health and

Human Sciences, Macquarie University, Sydney, Australia

${ }^{2}$ Department of Practice and Policy, UCL School of Pharmacy, University

College London, BMA House, Entrance A, Tavistock Square, Bloomsbury, London WC1H 9JP, UK

Full list of author information is available at the end of the article
}

(c) The Author(s). 2020 Open Access This article is licensed under a Creative Commons Attribution 4.0 International License, which permits use, sharing, adaptation, distribution and reproduction in any medium or format, as long as you give appropriate credit to the original author(s) and the source, provide a link to the Creative Commons licence, and indicate if changes were made. The images or other third party material in this article are included in the article's Creative Commons licence, unless indicated otherwise in a credit line to the material. If material is not included in the article's Creative Commons licence and your intended use is not permitted by statutory regulation or exceeds the permitted use, you will need to obtain permission directly from the copyright holder. To view a copy of this licence, visit http://creativecommons.org/licenses/by/4.0/ The Creative Commons Public Domain Dedication waiver (http://creativecommons.org/publicdomain/zero/1.0/) applies to the data made available in this article, unless otherwise stated in a credit line to the data. 
(Continued from previous page)

Conclusions: Within oncology, CPOE appears to move the 'mix' of risk strategies towards ultra-safe models of safety and protocol-mandated care. However, in order to operate ultra-safe strategies embedded in CPOE and stay on protocol it is essential for clinicians to be thoughtful and show sensitivity to operations in CPOE Use. CPOE design can be advanced by better consideration of mechanisms to support interdependencies.

Keywords: Health information technology, Evaluation, Medication safety, System safety, Paediatrics, Hospitals, Human factors, Qualitative research

\section{Background}

Chemotherapy medications are hazardous, high risk treatments; errors with these medications can cause severe harm, especially in children [1]. Chemotherapy regimens are administered often in combination with other medications and over many months, and errors are often associated with interdependent elements of chemotherapy regimens, such as number of cycles, dose scheduling, cumulative doses, and monitoring $[1,2]$.

To reduce harm from chemotherapy, risk management strategies must be in place [3], individual patient regimens must be based on documented and referenced protocols [4], and work processes organised with reference to accepted standards [4-7] and designed to reduce the possibility of error $[8,9]$. Computerized provider order entry (CPOE) systems with decision support are a further strategy for improving safety with chemotherapy. CPOE can reduce medication errors and unwanted protocol deviations by automatically calculating chemotherapy doses based on patient height and weight, providing warnings, assisting in scheduling of chemotherapy cycles, and supporting workflow [10-16]. Conversely, CPOE can introduce new types of error and have unexpected negative consequences on work practices [1, 17]. It can also be challenging to implement CPOE for chemotherapy $[15,16,18,19]$, with every chemotherapy protocol having to be entered in the system and then kept up to date $[15,20]$. Adoption of CPOE for chemotherapy can thus lag behind adoption of CPOE systems for other medications or in other clinical areas [15].

The literature suggests that design of CPOE for chemotherapy is 'confronted with problems' [21] and that CPOE alone is not sufficient to eliminate chemotherapy errors. Other safety strategies generally co-exist, including error surveillance systems [22, 23], checking of patient regimens against standard protocols [22, 24], and interventions to improve situational awareness [12, 22]. The literature remains unclear on how CPOE relates to such strategies, especially with regard to management of interdependencies. This gap in knowledge may hinder efforts to improve CPOE design and implementation, and thus improve patient safety.

This study aimed to fill this gap by investigating how CPOE for chemotherapy relates to other safety strategies in use in a paediatric clinical oncology unit, with a focus on the management of interdependencies. Our objectives were to identify the strategies clinicians apply to safely manage the interdependencies inherent in paediatric chemotherapy, and whether/how CPOE affects these.

For this study, we drew on theoretical and practical insights from Vincent and Amalberti's research on safety strategies 'in the real world', and the three main safety models they propose - ultra-safe, high-reliability organisations (HROs) and ultra-adaptive [3, 25, 26]. We briefly explain their framework in the next section, as we describe our methods and provide definitions.

\section{Methods \\ Analytical framework}

Vincent and Amalberti [3] explain the different 'real world' safety strategies applied in different sectors or organisations for dealing with risks. Such strategies may involve, for example: 1 . eliminating exposure to risk by defining 'no go' contexts for operations (following Amalberti, we refer to this as 'Plan A' [25] - in aviation, equivalent to grounding all flights when a volcano erupts) and applying rigid standard operating procedures; 2. introducing barriers to risks by engineering optimisation of work processes (including by introducing technology); 3. dealing with risks by improving organisational capacity for monitoring, adaptation and response (e.g. by maintaining 'sensitivity to operations' [27], or awareness of how one's actions affect others); and 4. developing professional expertise for extreme situations. We considered these strategies as different ways, at different levels, to manage risks associated with interdependencies. These strategies also approximately correspond to three models of safety in organisations (Fig. 1) - the ultra-safe (examples 1 and 2 above), high reliability organisation (HRO) (example 3) and ultraadaptive (example 4) [3, 25]. Different healthcare contexts have a different combination of the three models, with some working more often than others as ultra-safe, HRO, and/or ultra-adaptive, along a continuum. Thus, it may be expected that: 'A healthcare team might, in one afternoon, work in an ultra-safe manner at some points, such as when a care pathway is clearly defined and entirely appropriate for the patient; they may work in a 


\begin{tabular}{|c|c|}
\hline $\begin{array}{l}\text { Main characteristics } \\
\text { and strategies }\end{array}$ & $\begin{array}{l}\text { Examples outside health } \\
\text { care and within health care }\end{array}$ \\
\hline \multicolumn{2}{|c|}{ Ultra-safe - avoiding risk } \\
\hline $\begin{array}{l}\text { Power to regulators and supervision of the system } \\
\text { to avoid exposing front-line actors } \\
\text { to unnecessary risks } \\
\text { Strategies include: defining 'no go' contexts for } \\
\text { operations and applying rigid standard operating } \\
\text { procedures; optimisation of work } \\
\text { processes/automation }\end{array}$ & $\begin{array}{l}\text { Civil aviation | } \\
\text { Nuclear Industry } \\
\text { Radiotherapy } \\
\text { Blood transfusion }\end{array}$ \\
\hline \multicolumn{2}{|c|}{ High reliability - managing risk } \\
\hline $\begin{array}{c}\text { Power to the group to organise itself, } \\
\text { apply procedures, adapt and make sense } \\
\text { of the environment } \\
\text { Strategies include: organisational learning, } \\
\text { improving organisational capacity for } \\
\text { monitoring, adaptation and response, learning } \\
\text { from incidents } \\
\text { - 'collective mindfulness' }\end{array}$ & $\begin{array}{c}\text { Firefighting | } \\
\text { Drilling industry } \\
\text { Scheduled surgery } \\
\text { Chronic care }\end{array}$ \\
\hline \multicolumn{2}{|c|}{ Ultra-adaptive - embracing risk } \\
\hline $\begin{array}{c}\text { Power to experts to rely on personal resilience, } \\
\text { expertise and technology to survive and prosper } \\
\text { in adverse conditions } \\
\text { Strategies include: developing professional } \\
\text { expertise, knowing one's own limitations, } \\
\text { learning from successes }\end{array}$ & $\begin{array}{l}\text { Himalaya mountaineering | } \\
\text { Professional fishing } \\
\text { Innovative medicine } \\
\text { Trauma centres }\end{array}$ \\
\hline
\end{tabular}

Fig. 1 Three models of safety and examples of associated risk strategies. Elaboration of Vincent and Amalberti text and figures [3, 25], with additional reference to collective mindfulness - typical of high reliability organisations models of safety [27]. Collective mindfulness manifests when people on the frontline collectively show preoccupation with failure (ongoing wariness that errors are possible), reluctance to simplify interpretations of unexpected events (questioning assumptions, uncovering blind spots), sensitivity to operations (having an integrated understanding of current situation, e.g. awareness of how one's actions affect others), commitment to resilience (awareness that it is impossible to anticipate all situations, needs for adaptation) and deference to expertise (persons with expertise to make decisions regardless of hierarchy) [27]

high-reliability mode for the main part and, for short periods, in an ultra-adaptive mode.' [3]

Building on Thompson's Organization in Action [28], we define interdependency as the relation between tasks or activities, where related tasks/activities must all be completed in a timely fashion, reliably, and/or safely, to achieve safe processes and/or outcomes. While many interdependencies are sequential (one task contributing to the next; a task cannot be completed unless other tasks have been completed first), others may be reciprocal (one task contributing to the other and vice-versa), or pooled (each activity independently contributing to the whole) [28]. For example, nurses' chemotherapy administration depends on doctors' prescribing, which may depend on pathologists' reporting results (sequential tasks); supplying medications in hospital pharmacies on a just-in-case basis, contributes to doctors prescribing medications to patients, while doctors prescribing also calls for medications being stored in pharmacy on a just-in-case basis (reciprocal relationship between activities); repeated administration of chemotherapy doses to a patient cumulates towards a safe maximum level (pooled activities). We define time dependencies as those where safety in management of the interdependency requires attention to timeliness or timing of related tasks (e.g. administration of sequential doses at specific time intervals). 


\section{Setting and design}

The study took place in the oncology unit of a 350-bed tertiary paediatric hospital in New South Wales (Australia) between September 2018 and June 2019. The oncology unit is the largest children's cancer unit in the State, with up to 150 new referrals each year. The unit has an inpatient ward plus an outpatient clinic run as an 'open door' service where patients and their families can be seen without an appointment. Doctors are routinely called to see patients not under their regular care.

Eighteen months prior to this study, the hospital had implemented hospital-wide CPOE (Cerner [29]) for prescribing and administration of medications, test orders and results, as part of an electronic medical record system. The oncology unit made use of CPOE functionality for linking orders ('PowerPlan') to incorporate chemotherapy regimens into the system and to prescribe and administer chemotherapy for individual patients. A chemotherapy PowerPlan team was responsible for building chemotherapy protocol templates in the CPOE and training oncology clinicians in use of the system.

We carried out a qualitative, interpretative study combining incident report analysis and interviews. Analysis of the incident reports informed the interview questions, and the insights gained through the interviews fed back to further analysis of the events described in the incident reports.

\section{Data collection}

Incident reports were submitted by staff through the hospital online incident reporting system. Any event that resulted in (or had potential to lead to) injury, damage or other loss is required to be reported. The hospital provided us with all incident reports concerning oncology patients recorded in the period 15 August 2016 to 15 February 2018 (the first 18 months following CPOE implementation in oncology), whether or not these were specifically related to medications or CPOE.

Interviews were carried out by a researcher (VL) with a background in health informatics and qualitative research. All clinicians in the oncology unit and members of the chemotherapy PowerPlan team were invited to participate as we were seeking to maximise variety of levels of seniority and CPOE expertise. Access to participants was facilitated by the unit coordinator. We sought participants for interviews until we reached code and meaning saturation [30]. Interviews were semistructured, using questions (Additional file 1) aimed to uncover how clinicians deal with interdependencies in the medication process, and whether the CPOE supported their work. With participants' written consent, interviews were audio recorded and professionally transcribed.

\section{Data analysis}

We carried out a qualitative content analysis [31] of the text narratives of the incident reports (fields for Incident description and Contributing factors) and the interview transcripts. Analysis was carried out with support of NVivo v.11, by one researcher in discussion with coinvestigators. The free text fields from incident reports were treated as narrative accounts of events from frontline clinicians [32, 33]. All reports were included, but only those related to direct patient care coded. We took both a conventional (inductive) content analysis approach, with open codes derived from the data, and a directed (deductive) approach with our objectives providing higher level categories for grouping the open codes.

Analysis of interview transcripts was carried out iteratively. First, an in-depth detailed understanding of the data was gained through careful reading and line by line coding. Open codes were applied through both conventional and directed approaches, to address study objectives and capture significant aspects of the context. Chemotherapy medication processes, as described by clinicians in the unit, were represented in flowcharts to better understand interdependencies in workflow. This analysis was done concurrently with, and soon after, data collection. A framework analysis approach [34] was then applied using categories from Vincent and Amalberti's model.

Throughout the analysis process, discrepant findings were sought. Findings from incident analysis were triangulated with those from interviews to enrich understanding [35]. The head of the oncology unit (LDP) checked 'the "fit" between respondents' views and the researcher's representation of them' [36] (credibility).

\section{Results}

A dataset of 827 incidents were received from the hospital and included in the analysis. Incidents not concerning direct patient care were then excluded $(n=73)$. (Details of the 827 incidents are given in Additional file 2 and elsewhere [37]). In the analysis of the incidents, the CPOE appeared to be both a mechanism for safety and a contributing factor for incidents.

Twenty interviews were conducted with 19 participants (Table 1). Interviews lasted about $30 \mathrm{~min}$.

We integrated results from interviews and incidents analysis and structured these around interdependencies and risks to medication safety, strategies to deal with these risks, and the role of the CPOE for each strategy. We identified quotes from interview transcripts by interview number (idX), but do not provide details on interviewees' roles to protect their anonymity. We refer to incident reports by their row in the dataset (iX). 
Table 1 Interview participants

\begin{tabular}{|c|c|}
\hline Role & $\mathrm{n}$ \\
\hline Consultant oncologists $^{a}$ & 2 \\
\hline Fellows & 4 \\
\hline Registrars & 3 \\
\hline Senior resident medical officer & 1 \\
\hline Specialist pharmacist & 1 \\
\hline Nurse unit manager ${ }^{\mathrm{b}}$ & 1 \\
\hline Registered nurses ${ }^{b}$ & 4 \\
\hline Clinical nurse consultant & 1 \\
\hline Information technology team - oncology pharmacist background & 1 \\
\hline Information technology team - oncology nurse background & 1 \\
\hline Total & 19 \\
\hline
\end{tabular}

a one interviewed twice; ${ }^{\mathrm{b}}$ within the same group interview. Paediatric oncology fellows and registrars in New South Wales (Australia), are resident doctors with oncology as their speciality, considered 'consultants in waiting' and 'consultants in training' respectively. This notation may be different internationally

Interdependencies and risks to safety with chemotherapy Work practices related to paediatric chemotherapy treatments were rich in interdependencies. Across interviews and incidents, we identified two interrelated overarching types of interdependencies that characterised chemotherapy prescribing and administration: first, those related to the organisation of clinical activities, in particular the medication workflow involving doctors, nurses and pharmacy staff, and its interplay with a variety of hospital services (e.g. intensive care, pathology, imaging, as well as other hospitals), and second, interdependencies inherent to chemotherapy regimens that dictated combinations of medications and tests. Time dependencies were especially apparent, such as precise time gaps between administration of successive doses.

Both types of interdependencies (organisational and regimen-related) were identified in incident reports and often involved CPOE. For example, an incorrect date in a CPOE prescription and a delay in documentation of the patient being 'ready for chemotherapy' (organisational dependency-medication workflow) delayed administration due to the medication requiring pre-medication (a regimen dependency), which further cascaded into requiring monitoring of the patient 'after hours', leading to increased risks for the patient and costs to the hospital (organisational dependency).

\section{[...] Patient due rituximab and MTX [methotrexate]} today, date for chemotherapy was incorrect and not 'ready for chemotherapy' documented [in the CPOE]. This delayed chemotherapy administration until 11am. [...] [medications] will need to be reordered. The first medication requires premedication and the patient is at risk of anaphylaxis with this drug (needs to be given in business hours) and MTX requires blood monitoring levels and if given outside set hours adds significant cost [...] [i150]

\section{Ultra-safe risk strategies applied to chemotherapy processes}

Chemotherapy safety risks were reported to be managed through a range of strategies based on the application of rules and multiple safety checks. These appeared more typical of the ultra-safe model of safety [3] than the HRO model.

Specifically, participants spoke of rigid rules regarding who was authorised to prescribe chemotherapy (on CPOE) and the content of medication orders as per chemotherapy protocols. Continuing with a regimen (e.g. progressing to the next cycle) was organised around the principle of withholding treatment until the patient recovers (comparable to 'Plan A' in the ultra-safe model).

Participants reported multiple checks during the medication process. In particular, nurses were tasked with checking each dose against the protocol to alert doctors to any discrepancy. This was 'an institution specific practice' (id16) that was maintained after CPOE implementation, despite the use of pre-set CPOE templates that would (or should) support doctors to produce orders matching the corresponding protocol.

...when I am ordering it [on CPOE], it looks fine to me. [But] The nurses [would] say, "Well, can't do that because it is out of sequence," or, "you have pushed this onto that." [...] Or they will say, "The protocol mandates $120 \%$ of this and you are giving $110 \%$ or $150 \%$." And they are just simply saying, "Is this what you want me to do?”... (id16)

Sometimes the patient's condition led to clinicians deciding to deviate from the protocol - such as when 'this child has such a high-risk disease that no matter what I'm going to go ahead' (id16) despite test results not reaching the threshold indicated by the protocol. This kind of judgement - highly dependent on individual clinical expertise - typically belongs to the ultra-adaptive model of safety. However, in this context - where the doctor had to communicate the decision to the nurses and to other doctors in order to act on it - it is perhaps suggestive of a HRO approach to dealing with risks.

\section{Does CPOE support an ultra-safe model of safety with chemotherapy?}

CPOE supported strategies typical of the ultra-safe model through hard and soft mechanisms, namely through automation, access to information and standardisation of the semantics of protocols (disambiguation) (Table 2). 
Table 2 Ultra-safe strategies to avoid medication errors/unwanted protocols' variations: automation and 'soft' support

\begin{tabular}{lll}
\hline Risk strategy (ultra-safe model) & CPOE support & $\begin{array}{l}\text { Examples from the data of how the CPOE supports the use } \\
\text { of ultra-safe strategies (compared to paper-based systems) }\end{array}$ \\
$\begin{array}{l}\text { Treatment is independent of } \\
\text { specific clinicians }\end{array}$ & $\begin{array}{l}\text { Visibility and access to protocol rules } \\
\text { and a patient prescribed treatment }\end{array}$ & $\begin{array}{l}\text { [...] your treatment has to go ahead whether I'm alive, dead, } \\
\text { overseas [...] someone else has to bear the brunt of knowing } \\
\text { where they're at. So it's really crucial for us to have a system } \\
\text { whereby the - where they're at' is easily accessible, (idi) }\end{array}$
\end{tabular}

Everyone knows/has access to Visibility and access to protocol rules the rules and a patient prescribed treatment
Standardised protocols across patients and clinicians

Rules on the content of the prescription (as per protocol)

Rules on who can act on the prescription

Treatment stopped until patient recovers ['Plan A']

Checks along the process

Checks against the rules
Disambiguation of protocol rules

CPOE templates, with linked pre-set orders

Automatic calculations by embedded rules

Warnings about breaking rules
Users' profiles and workflow management systems to route the prescription

'Ready for chemo' checkbox no administration until 'ticked'

Double signatures required to unlock next step

Nurses verifying treatment based on access to the protocol ...we have got immediate access to what the protocol looks like and [...] to the documentation. [...] they're not squirreled away in some cupboard, they're not lost, they're available electronic everywhere. (id1) [nurses would] go to the orders page. [...] And they'll go okay, so he's in hospital [...], so he came in on Sunday, tomorrow he's due vincristine. I have to make sure I check with the doctors, whether they want him to go ahead with it or delay it, which they would never have known in advance, previously. They'd never have been able to see that. Because they didn't have any of their chemotherapy records when they got admitted. [...] Their visibility of it is probably one of the most important things of the plans. (id14)

... whether an or and a comma in a sentence meant one thing was inclusive of another, or one thing was exclusive of another. So even within protocols, even the smallest wording, [...] you've really got to sit down and discuss as a team what you think it means, ... (id13)

...depending on the drugs that go into [the CPOE plans] and how they relate to each other [...] they might [...] have a timed relationship to say you're not allowed to start drug $Y$ until drug $X$ has finished or run them both at the same time... (id13)

You're meant to give this over four hours, [...] - in paper, that was just written and then pharmacy would come along and, kind of, annotate it, whereas now it's prebuilt ... (id7)

all the drugs listed here, you just have to agree to it [...] [the CPOE system] will limit dose, [system says two] you can't order two and a half. So there are certain protections in the system... (id1)

... [the fellows] - they can sign it but it doesn't go to pharmacy until it's [gone] to the oncologist. [...] Once [the oncologist] reviewed it, it goes to pharmacy. [...] [Pharmacy] can't make it up until [it's been reviewed by the oncologist]. (id1)

...there is a flow that says, well say it's more than 30 white cells, [...], liver function is less than that, [...] very clear criteria and then only if you tick them and if they are okay will it allow you to go ahead and sign for a go ahead. (id2)

....we are much more rigid in terms of two people checking, two people going to the bedside. (id8-12)

... [before CPOE] you had to have the paper protocol [...] [with the CPOE] the nurses [...] [have] access to the protocol. So, when they saw a particular dose scheduled, they would go to the protocol, check it and say 'yes'. And, [...] if it didn't marry, then they would report it as an incident ... (id16)
In terms of automation, the CPOE provided clinicians with all required medications pre-built in templates, to reduce the possibility of medications being omitted by mistake, which were also linked with appropriate time dependencies (if doctors delayed one, the others would be automatically delayed for a corresponding period). The CPOE provided automatic dose calculations and warnings based on embedded rules, and 'exposed' unreliable patient weight and height data used for dose calculation, by displaying trends and normal ranges.

... you can see patients with a weight here and a height up here [on the CPOE chart] [...] three months for a baby of four kilos you can go to eight kilos, you can change dramatically the dose. [...]. Now, in paper land [...] [doctors] might go six months without checking the height and weight. It happened before, but here it's exposing it. (id1)

CPOE automation also limited prescribing and administration rights through different users' profiles; the embedded workflow management system automatically routed the orders to the authorised person. The CPOE paused the workflow proceeding forward to administration until the system had been notified via a checkbox that the patient was 'ready for chemo'.

However, these CPOE contributions towards an ultrasafe approach to chemotherapy were challenged by the 
perceived complexity of operating the system (especially when protocol templates were not available in the system to prescribe a patient's regimen), and difficulties in identifying 'where the patient is at' in the protocol.

In terms of 'soft' mechanisms, we identified two aspects of CPOE that supported use of ultra-safe risk strategies. All clinicians had access both to patient information and the relevant chemotherapy protocol; previously, paper-based protocols were kept in drawers and not easily accessible across place and time. In addition, the process of converting paper-based protocols into electronic versions brought to the surface previously invisible ambiguities in their rules. To automate these rules, these had to be clarified and uniquely defined so that all clinicians would interpret the protocol in the same way.

HRO strategies - management of uncertainties and 'usual hazards' through adaptation and different time horizons The application of the principle of withholding treatment until the patient recovers ('plan A') meant that planned doses or cycles often needed to be rescheduled. Thus, for any patient there would be uncertainty about when exactly the treatment would be given. This scheduling - or rescheduling - of the treatment must also take into account the constraints posed by the hospital's resources (organisational interdependencies), for example 'our general anaesthetic days are Monday and Thursday' (id16), or over holidays, 'services aren't working quite as well' (id1). The patient and family may also pose constraints on suitable dates - such as wanting to avoid school photo day.

Clinicians reported that it was challenging to plan cycles, tests and doses much in advance. Although the CPOE theoretically allowed clinicians to prescribe a whole regimen in advance, given these uncertainties, interviewees indicated that they would only schedule about a month ahead in practice.

Researcher: the doctors might schedule the entire one year of treatment in advance? Participant: [...] they probably wouldn't. [...] we would chart chemotherapy, kind of, a month out, because [children] can grow so much in such a short time, the doses need to change. Other things can happen as well that they may start to not recover as well, so we need to modify the dose, it was too big for them, so we need to back off [...]. (id7)

Doctors therefore made use of time as a risk management strategy [25] to deal with the uncertainties. More specifically, we identified three time horizons used to approach a patient's chemotherapy regimen - a life-long view, the whole regimen view, and the 'here and now'.
CPOE automation better supported the 'here and now' than the longer time views (Table 3). For example, clinicians reported difficulties with finding and collating information to calculate a patient cumulative dose (a lifelong view), and with the fragmented electronic display of the whole regimen.

\section{Sensitivity to operations and CPOE}

We found that a combination of situation awareness, organisational awareness and CPOE awareness was perceived as essential for safe and efficient CPOE use (Fig. 2). Clinicians had to maintain (and act on) situational awareness to understand 'where is the patient at' in their specific regimen and with respect to the corresponding protocol. Several interviewees referred to this information need - 'where patients are up to' (id1, id5, id7, id8-12). Clinicians also had to maintain (and act on) organisational awareness. For example, they had to act on the system with awareness of self and others 'down the line' (Table 4). They had to be aware of CPOE, learning to be watchful of its automated behaviour such as automated recording of times and dates. With use, clinicians learned that, for a variety of reasons, time and dates in the system may not accurately reflect the times when medication activities took place. This had repercussions for regimen time dependencies, which were also encoded in the templates.

...sometimes when [the CPOE] says, it's the start date, it's not really been the start date, because it's been delayed [...]. That stays as the estimated start date [...] when you come to [prescribe], they're due next week, for example, but if you look at the chemotherapy, [...], they're due in two weeks. [...] just [automatically] ticks over [...] (id3)

\section{Discussion}

We studied a paediatric oncology unit using a hospitalwide CPOE system, and identified two inter-related overarching types of interdependencies associated with chemotherapy: those related to the organisation of clinical activities, and those inherent to chemotherapy regimens in terms of dictating precise combinations of medications, and combinations of medications and tests. Time dependencies were especially apparent across both interdependency types. The time dependencies in a patient regimen were dealt with through a cognitive strategy known as 'fragmentation' ('allowing shorter horizon planning' [39]) and a practice of temporary plans and constant adjustments. These we associate with 'adaptation' strategies typical of HRO models of safety.

CPOE automation seemed to better support the small scale, short-time regimen dependencies that are largely 
Table 3 Doctors' time horizons over chemotherapy treatments and computerised provider order entry (CPOE) support

\begin{tabular}{|c|c|c|c|}
\hline Time horizon $^{\text {a }}$ & $\begin{array}{l}\text { Uncertainty and/or risks associated } \\
\text { with interdependencies managed } \\
\text { with this time horizon }\end{array}$ & $\begin{array}{l}\text { Examples of how CPOE supports } \\
\text { this (or not)* }\end{array}$ & Examples from the data \\
\hline life-long & $\begin{array}{l}\text { A view over cumulative effects } \\
\text { of medications over time, to } \\
\text { manage life-long risks (e.g. hearth } \\
\text { failure) }\end{array}$ & $\begin{array}{l}\text { Compared to paper-based records, } \\
\text { CPOE makes patient information } \\
\text { available, independent of time and } \\
\text { place } \\
\left(^{*}\right) \text { Difficulty in tracing specific data } \\
\text { across different orders/screens } \\
\left(^{*}\right) \text { IT teams can manually build into } \\
\text { the system automated alerts on } \\
\text { cumulative doses - but this must be } \\
\text { done for each drug, and may not be } \\
\text { available when needed }\end{array}$ & $\begin{array}{l}\text {...the cardiologist team always need to } \\
\text { know how much anthracyclines they've } \\
\text { received, because it's cardiotoxic and the } \\
\text { dose that they've received makes a } \\
\text { difference about what was risk stratifying } \\
\text { and things. So to try and find a patient's } \\
\text { cumulative anthracycline dose sometimes } \\
\text { has been exhausting. More exhausting } \\
\text { than night shifts exhausting. (id20) }\end{array}$ \\
\hline whole treatment & $\begin{array}{l}\text { A view over 'where we are at' in the } \\
\text { protocol as a whole; how the } \\
\text { protocol is being given - possibly } \\
\text { adapted - to the patient, and the } \\
\text { patient response to treatment. It is } \\
\text { a view of how much chemotherapy } \\
\text { overall is needed, to minimise } \\
\text { toxicity and maximise effect, and } \\
\text { how it is given over time }\end{array}$ & 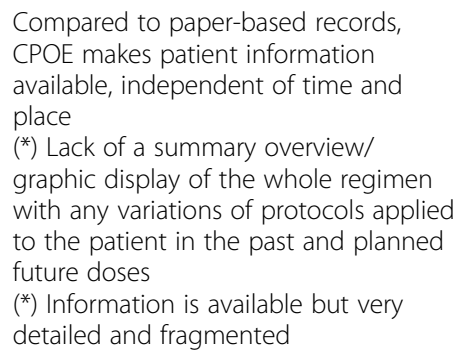 & $\begin{array}{l}\text { I can go through a three-day admission, } \\
\text { it's all there in a capsule. But when that } \\
\text { becomes } 6 \text { months or } 12 \text { months or } \\
5 \text { years, ... the system is not geared to } \\
\text { allow us to navigate to the critical } \\
\text { information [...] The treatment is listed } \\
\text { as a series of lines [...] it's very hard to } \\
\text { actually visualise that they haven't got } \\
\text { an extra dose at day } 15 \text { or they've } \\
\text { missed a dose at day seven, ... (id6) }\end{array}$ \\
\hline here and now & $\begin{array}{l}\text { A view over the requirements and } \\
\text { constraints for the administration } \\
\text { of the current dose, to prevent } \\
\text { medication errors, unwanted } \\
\text { deviations from protocols, and } \\
\text { delays }\end{array}$ & $\begin{array}{l}\text { Automated pre-coded prescription } \\
\text { items, time dependencies and } \\
\text { scheduling, all items included in } \\
\text { the prescription at planning stage }\end{array}$ & $\begin{array}{l}\text {...the main thing is the system is built on } \\
\text { protocol. [...] it follows almost exactly the } \\
\text { way the protocol is written, almost all the } \\
\text { time. And if in case what I am prescribing } \\
\text { is a deviation from what is allowed, then [...] } \\
\text { it is going to flag up. For example, if something } \\
\text { that I needed to be charted for day } 1, \text { I charted } \\
\text { again for day } 2 \text { and } 3 \text { [..], then it will say, it's } \\
\text { already there why are you [ordering] it? [...] } \\
\text { and it's really handy that [...] the cut offs } \\
\text { [safety thresholds] are already there, right next } \\
\text { to that. (id2) } \\
\text { the timing [...] often it's pre-suggested as well, } \\
\text { depending on what medication you're charting } \\
\text { it will already have a suggested frequency that } \\
\text { you should be charting it (d5) }\end{array}$ \\
\hline
\end{tabular}

${ }^{a}$ Over a life-long view, there are concerns for life-long risks of cumulative doses. A whole treatment view is required for most treatment decisions; each dose is assessed in view of patient's response to previous doses, in relation to future doses, future options for treatment - at any point in time, the sensemaking is retrospective and prospective. The view over the here and now is concerned with 'the minutiae' of the medication process related to the specific dose, around the time of administration. It's where the order is finalised, multiple checks performed, medications given (or not) - a time that may span over a few days

under control of the team, rather than the scheduling of services where organisational dependencies are not under their control. It is possible that the small scale, short-time regimen dependencies were mainly sequential interdependencies (e.g. administration of different medications as sequential steps), while the organisational dependencies were also relational or pooled, and that sequential interdependencies are easier to automate in workflow management systems.

Both ultra-safe and HRO strategies were used to address chemotherapy risks and interdependencies. CPOE automation supported application of ultra-safe strategies in chemotherapy such as compliance with protocols' rules and 'no-go' contexts for proceeding with chemotherapy. CPOE support mechanisms for an ultra-safe model of care were both 'hard' (automation) and 'soft' (information availability/disambiguation). However, prescribing regimens with CPOE was difficult when protocol templates were not readily available; it was difficult to track medication data across screens, and a summary display of the whole regimen (showing protocol variations, if any) was not available; automated time-stamps of medication administration were not always accurate, with repercussions for subsequent time-dependent doses. Therefore, in addition to situational awareness at the level of the patient [38] and awareness of the organisation's teams and services [27], clinicians had to maintain awareness of the technology ('CPOE awareness'), its limitations and consequences. We propose a definition of CPOE awareness as knowledge of how the technology works, how to make it work, and the effects it produces, and acting on this knowledge. 


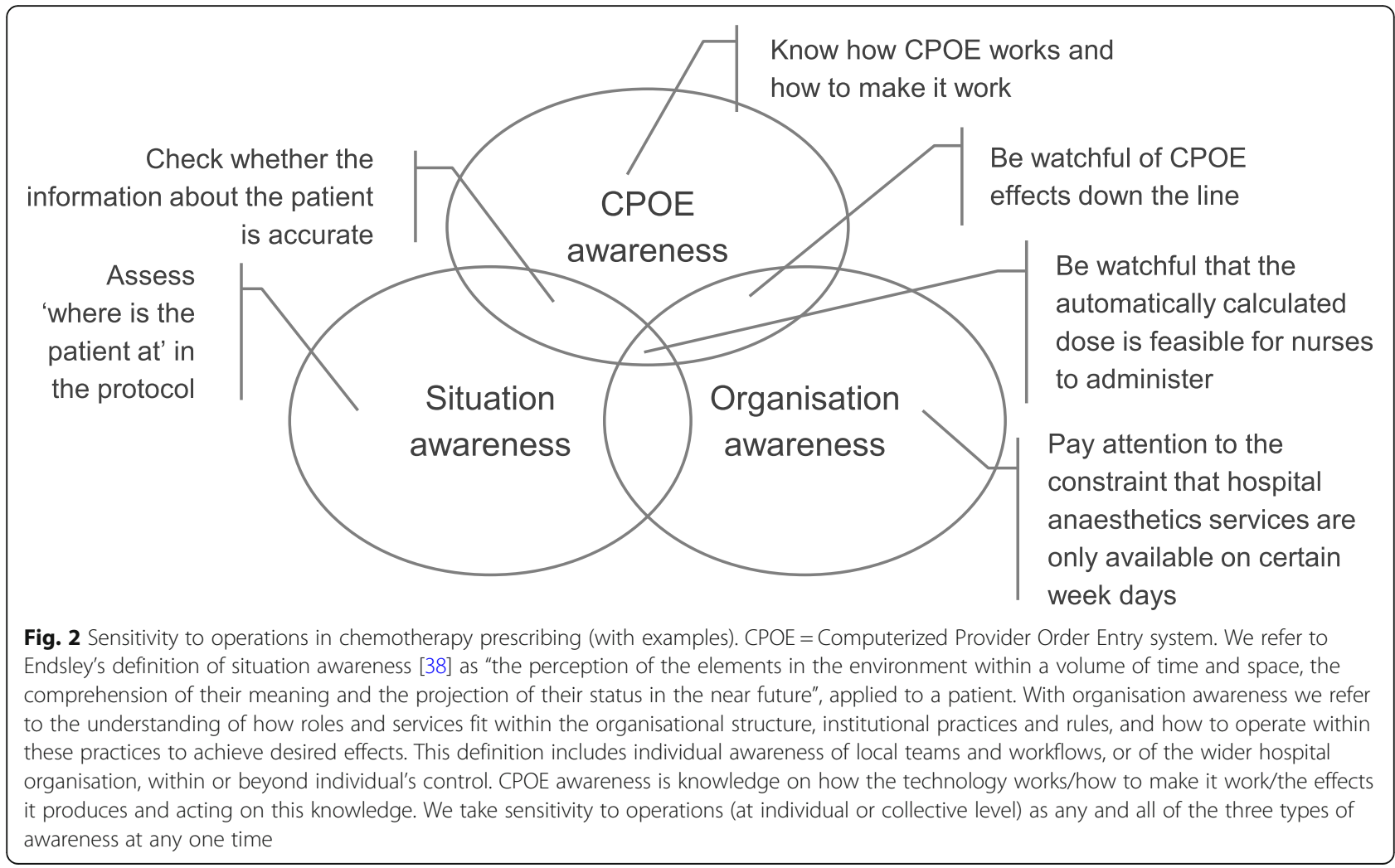

The finding that 'CPOE awareness' was needed in this setting is consistent with other CPOE chemotherapy implementations that required significant investment of time and resources to enable clinicians to gain necessary familiarity with all the nuances of CPOE workflows [19]. Our finding that CPOE automation supported application of ultra-safe strategies in chemotherapy is in line with existing literature that shows how CPOE may improve compliance with chemotherapy protocols [17]. However, prescribed regimens are subject to change and in need of adaptation, also due to application of ultrasafe rules (typically, waiting for patient recovery before proceeding with treatment). In the late 1990s, medical sociologists Timmermans and Berg explained how standardisation of patient care with (paper-based) oncology protocols was achieved through clinicians' 'active (not mindless) support [...] to maintain the protocol's trajectory on course' [40]. In their account, adaptation was an enabler, rather than opposer, to standardisation. We posit that active (not mindless) adaptation requires situational awareness (knowledge of 'where is the patient $a t^{\prime}$ in relation to a prescribed regimen and underlying protocol), which must be facilitated by CPOE displaying interdependent elements of regimens over different time horizons (in chemotherapy, known as 'roadmaps' [12]). This is also supported by recent research suggesting that chemotherapy errors with CPOE may be prevented with knowledge of a patient's chemotherapy history and in- depth knowledge of protocols [41]. Nurses' and/or pharmacists' routine checks of CPOE orders against protocols $[22,24]$ - also reported in the unit we studied - are one way to address CPOE limitations by automatically monitoring variations to protocols, and may also be facilitated by the availability of electronic roadmap displays.

Strengths of this study include multiple sources of data - interviews and incident reports - and analysis informed by theory. A limitation is that we did not observe the activities described. Instead, we relied on participants as 'their own ethnographers' [42]. We had limited nurse participation. We identified ultra-safe and HRO strategies; we did not find any strategy from the ultraadaptive safety model possibly because we did not observe activities. Patient safety incident reports were brief and provided only limited information; incidents related to multiple aspects of patient care, not exclusively chemotherapy. We took a qualitative approach to incident analysis given the limitations of analysing these quantitatively; reported incidents are likely to represent only a fraction of the incidents that occur and thus numbers of incidents cannot be relied on to establish frequency [43]. The CPOE system investigated was a general system adapted for chemotherapy, rather than a bespoke chemotherapy system; it is not known whether the findings generalise across other organisations and other systems. 
Table 4 Examples of (organisation) team level awareness and computerised provider order entry (CPOE) limitations

\begin{tabular}{ll}
\hline Team perspective & CPOE issue to be aware of \\
\hline Doctors with doctors & $\begin{array}{l}\text { Difficult to identify 'where the patient is } \\
\text { at' [whole regimen view] }\end{array}$
\end{tabular}

Difficult to identify [patient specific] changes to prescriptions

Doctors with nurses

Nurses with nurses

Doctors with Pharmacy
Difficult to identify 'where the patient is at' [exact place in the protocol (a PDF file)]

Automatically calculated doses may not be feasible to administer

Automatically calculated times of doses may not be convenient (safe?)

Fluid charts may not get automatically filled-in

Difficult to identify 'where the patient is at' [exact place in the protocol (a PDF file)]

\author{
Automatically calculated doses may \\ not be feasible to dispense \\ Pharmacy staff access to CPOE orders \\ not sufficient for timely dispensing
}

Examples from the data of awareness of the impact of the CPOE for the team

....you do have to then plough through a fair bit of information to figure out where they're at. So I'm at the mercy of the quality of the input of the previous doctor [...] If they put in [...] for example, "had day 72 coming on the 14th [of this month] for day 86," then it places me exactly where they're at. [...] if they put that in it makes my life easy. If they put in, "See again next scheduled visit", I don't know what that next scheduled visit is. And for my patients I might know, [...] I will see other people's patients [...] I have to then come up to speed with what that particular patient is on. (id1)

I know the things that make my life easier, so I will document these things whenever I see them. And some of the fellows that see them in clinic, because they were more recently registrars and recognise this issue, will do the same. So they will document clearly their medication list, and the changes that they've made. (id20)

Nurse 1: [...] when the fellows are ordering the chemo [...] it does help if they are actually very specific on where this kid is up to, like, exactly what cycle and everything they are up to.

Nurse 2: 'Course two, cycle three, page 59'.

Nurse 1: The more specific they [doctors] are,

the easier it is

for us to find it, yeah. (id8-12)

[as a doctor] I make sure that the dose isn't something that's ridiculous and going to be really hard for [nurses] to give. (id5)

I make sure that [nurses] can recognise if they need to give it now or if they can give it at a later date, like [...] the midnight doses. [...] whether the times that are coming up are convenient. (id5)

... just things like being aware that if they [the doctors] don't fill out things like durations and infuse over times correctly, it makes it much harder for the nurses to then complete their fluid balance charts correctly, because they've got to manually pull everything in, whereas if the doctors order it correctly it all prepopulates beautifully $f$ or them. (id13)

Nurse 1: To find the bit you actually want for the chemo you are giving, you might have to scroll through multiple pages if you are the first [nurse] to kind of ... find that cycle of chemo.

Nurse 2: Once you found it we write it down [for the next nurse]. (id8-12)

So not prescribing a dose that's $1.62 \mathrm{mg}$ because no pharmacist is ever going to be able to draw that up ... (id13)

We owe it to the pharmacy to give them as much notice as possible and the minimum [lead] time we have most recently put in is $48 \mathrm{~h}$. So the pharmacy has to know at least $48 \mathrm{~h}$ before, we're going to give the drug... (id2)
Ultra-safe services 'are highly standardized and rely heavily on automation and information technology' [3]. Implementation of CPOE in chemotherapy appears to be a move towards ultra-safe, but our findings suggest that CPOE design must be improved. In such a complex and high-risk setting, CPOE design should facilitate clinicians' decision-making processes, rather than add difficulties. Lessons can be learned for design of chemotherapy CPOE that better supports the management of interdependencies in regimens and workflows [21]. This might include affording a variety of visualisation displays over different time horizons and capturing more 
accurate timestamps of activities, tracking protocol variations and cumulative effects over time. CPOE implementations also need to support learning processes for clinicians to gain the awareness needed to use CPOE systems safely. For this, sufficient enabling resources (e.g. staff/patient ratio, CPOE training and assistance, quiet time for prescribing) must be in place at the point of roll-out and maintained throughout staff turnover [19].

To our knowledge, this is the first study to apply Vincent and Amalberti's models of safety and related risk strategies [3] to evaluate a technology implementation in a healthcare setting. The study has been able to identify mechanisms by which CPOEs can mitigate or exacerbate medication safety risks.

Patients and families may have different priorities or values about medication safety and compliance with rules, that may vary during the long period of chemotherapy treatments. Future research in paediatric oncology should investigate whether/how patients and families' priorities or values change the way clinicians use the CPOE for chemotherapy regimens, and how they approach the different time frames: the 'here and now', 'whole treatment' and 'life-long'. Further research with CPOE for chemotherapy should also investigate time dependencies in regimens in more detail - including how nurses manage administration times between doses and automated timestamping of activities.

\section{Conclusions}

CPOE appears to affect the 'mix' of risk strategies in place in an oncology unit. It can drive ultra-safe models of safety and protocol mandated care, but operating ultra-safe strategies embedded in the CPOE and staying on protocol also requires HRO strategies including 'sensitivity to operations' in CPOE use. CPOE implementations need to support the processes of learning required for clinicians to gain such collective awareness, and shortcomings in CPOE design must be addressed for it to fully contribute to the ultra-safe.

\section{Supplementary information}

Supplementary information accompanies this paper at https://doi.org/10. 1186/s12911-020-01212-z.

Additional file 1: Appendix 1: Interview guide.

Additional file 2. Incident analysis - methods and summary of findings.

\section{Abbreviations}

CPOE: Computerized provider order entry; HRO: High-reliability organisations

\section{Acknowledgements}

We are grateful to the hospital staff who gave their time to participate in this study.

\section{Authors' contributions}

$V L$ conceived the study, collected the data, lead data analysis and drafted the manuscript. LDP assisted with data collection. BDF, JW and LDP contributed to data analysis and to the writing of the paper. All authors read and approved the final manuscript.

\section{Authors' information}

$V L$ is currently researching the impact of technology for medications on collective mindfulness and medication safety. LDP is oncology specialist and head of the cancer centre in the hospital we studied.

\section{Funding}

This study received funding from the European Union's Horizon 2020 research and innovation programme under the Marie-Skłodowska Curie Grant Agreement number 740131. This paper represents independent research supported by the NIHR Imperial Patient Safety Translational Research Centre. The funding bodies had no role in the design of the study and collection, analysis, and interpretation of data and in writing the paper. The views expressed are those of the authors and not necessarily those of the NHS, the NIHR or the Department of Health and Care. The European Commission and its Research Executive Agency are not responsible for any use that may be made of the information this paper contains.

\section{Availability of data and materials}

The datasets generated and/or analysed during the current study are not publicly available. The hospital has not consented to the sharing of patient safety incidents data beyond the project team. Research participants did not consent to interview transcripts being made publicly available.

\section{Ethics approval and consent to participate}

The study received ethics approval from the hospital's human research ethics committee (Ref HREC/15/SCHN/370). Hospital incident reports were deidentified prior to review and analysis. Interview participants provided written informed consent. Data from interviews were anonymised at the point of data collection.

\section{Consent for publication}

Not applicable.

\section{Competing interests}

Professor Franklin supervises a PhD student who is part funded by Cerner, outside the submitted work. All other authors have no conflicts of interest to declare.

\section{Author details}

${ }^{1}$ Australian Institute of Health Innovation, Faculty of Medicine, Health and Human Sciences, Macquarie University, Sydney, Australia. ${ }^{2}$ Department of Practice and Policy, UCL School of Pharmacy, University College London, BMA House, Entrance A, Tavistock Square, Bloomsbury, London WC1H 9JP, UK. ${ }^{3}$ Centre for Medication Safety and Service Quality, Pharmacy Department, Imperial College Healthcare NHS Trust, London, UK. ${ }^{4}$ Cancer Centre for Children, The Children's Hospital at Westmead, Sydney, Australia.

Received: 29 April 2020 Accepted: 3 August 2020

Published online: 14 August 2020

\section{References}

1. Weingart SN, Zhang L, Sweeney M, Hassett M. Chemotherapy medication errors. Lancet Oncol. 2018;19(4):e191-e9.

2. Small MD, Barrett A, Price GM. The impact of computerized prescribing on error rate in a department of Oncology/hematology. J Oncol Pharm Pract. 2008;14(4):181-7.

3. Vincent C, Amalberti R. Safer healthcare: strategies for the real world. Cham: Springer International Publishing; 2016.

4. Carrington C, Stone L, Koczwara B, Searle C, Siderov J, Stevenson B, et al. The Clinical Oncological Society of Australia (COSA) guidelines for the safe prescribing, dispensing and administration of cancer chemotherapy. Asia Pacific J Clin Oncol. 2010;6(3):220-37.

5. Fani Pakdel A, Mousavi M, Roohani M, Elyasi S, Kooshyar M. 113P Chemotherapy administration safety standards for preventing medication 
errors and adverse drug reactions in patients with breast cancers. Ann Oncol. 2016;27(suppl_9).

6. Neuss MN, Gilmore TR, Belderson KM, Billett AL, Conti-Kalchik T, Harvey BE, et al. 2016 updated American Society of Clinical Oncology/Oncology Nursing Society chemotherapy administration safety standards, including standards for pediatric oncology. J Oncol Pract. 2016;12(12):1262-71.

7. Coccia PF, Pappo AS, Beaupin L, Borges VF, Borinstein SC, Chugh R, et al. Adolescent and young adult oncology, version 2.2018, NCCN clinical practice guidelines in oncology. J Natl Compr Cancer Netw. 2018;16(1):6697.

8. Pfeiffer Y, Gut SS, Schwappach DL. Medication safety in oncology care: mapping checking procedures from prescription to Administration of Chemotherapy. J Oncol Pract. 2018;14(4):e201-e10.

9. Franklin $B D$, Panesar SS, Vincent $C$, Donaldson LJ. Identifying systems failures in the pathway to a catastrophic event: an analysis of national incident report data relating to vinca alkaloids. BMJ Q Safety. 2014;23(9):765-72.

10. Ferranti JM, Horvath MM, Jansen J, Schellenberger P, Brown T, DeRienzo $\mathrm{CM}$, et al. Using a computerized provider order entry system to meet the unique prescribing needs of children: description of an advanced dosing model. BMC Med Inform Decis Mak. 2011;11(1):14.

11. Gandhi S, Tyono I, Pasetka M, Trudeau M. Evaluating an oncology systemic therapy computerized physician order entry system using international guidelines. J Oncol Pract. 2014;10(2):e14-25.

12. Allen SW, Hayashi RJ, Jones SJ, Drozda MH, Brown RL, Lackey IT, et al. Development of electronic chemotherapy roadmaps for pediatric oncology patients. J Pediatr Oncol Nurs. 2018;35(5):314-9.

13. Elsaid K, Truong T, Monckeberg M, McCarthy H, Butera J, Collins C. Impact of electronic chemotherapy order forms on prescribing errors at an urban medical center: results from an interrupted time-series analysis. Int J Q Health Care. 2013;25(6):656-63.

14. Hoffman JM, Baker DK, Howard SC, Laver JH, Shenep JL. Safe and Successful Implementation of CPOE for Chemotherapy at a Children's Cancer Center. J Nat Compr Canc Netw. 2011;9(Suppl 3):S-36-50.

15. Martin DB, Kaemingk D, Frieze D, Hendrie P, Payne TH. Safe implementation of computerized provider order entry for adult oncology. Appl Clin Inform. 2015;6(4):638-49.

16. Whalen K, Lynch E, Moawad I, John T, Lozowski D, Cummings BM. Transition to a new electronic health record and pediatric medication safety: lessons learned in pediatrics within a large academic health system. J Am Med Inform Assoc. 2018;25(7):848-54

17. Rahimi R, Moghaddasi H, Rafsanjani KA, Bahoush G, Kazemi A. Effects of chemotherapy prescription clinical decision-support systems on the chemotherapy process: a systematic review. Int J Med Inform. 2019;122:206.

18. Wagner S, Beckmann MW, Wullich B, Seggewies C, Ries M, Bürkle T, et al. Analysis and classification of oncology activities on the way to workflow based single source documentation in clinical information systems. BMC Med Inform Decis Mak. 2015;15(1):107.

19. Chung C, Patel S, Lee R, Fu L, Reilly S, Ho T, et al. Implementation of an integrated computerized prescriber order-entry system for chemotherapy in a multisite safety-net health system. Am J Health Syst Pharm. 2018;75(6): 398-406.

20. Crespo A, Redwood E, Vu K, Kukreti V. Improving the safety and quality of systemic treatment regimens in computerized prescriber order entry systems. J Oncol Pract. 2018:14(6):e393-402.

21. Rahimi R, Kazemi A, Moghaddasi H, Rafsanjani KA, Bahoush G. Specifications of computerized provider order entry and clinical decision support systems for cancer patients undergoing chemotherapy: a systematic review. Chemotherapy. 2018;63:162-71.

22. Weiss BD, Scott M, Demmel K, Kotagal UR, Perentesis JP, Walsh KE, Significant and sustained reduction in chemotherapy errors through improvement science. J Oncol Pract. 2017;13(4):e329-e36.

23. Call RJ, Burlison JD, Robertson JJ, Scott JR, Baker DK, Rossi MG, et al. Adverse drug event detection in pediatric oncology and hematology patients: using medication triggers to identify patient harm in a specialized pediatric patient population. J Pediatr. 2014;165(3):447-52.e4.

24. Baldwin A, Rodriguez ES. Improving patient safety with error identification in chemotherapy orders by verification nurses. Clin J Oncol Nurs. 2016;20(1): $59-65$.

25. Amalberti R. Navigating safety: necessary compromises and trade-offs theory and practice: springer; 2013.
26. Amalberti $R$, Vincent $C$. Managing risk in hazardous conditions: improvisation is not enough. BMJ Q Safety. 2019; Published Online First: 09 July 2019:bmjgs-2019-009443.

27. Weick KE, Sutcliffe KM, Obstfeld D. Organizing for High Reliability: Processes of Collective Mindfulness. In: Sutton RS, Staw BM, editors. Research in Organizational Behavior. Volume 1. Stanford: Jai Press; 1999. p. 81-123.

28. Thompson JD. Organizations in action: social science bases of administrative theory: McGraw Hill; 1967.

29. Cerner Corporation: https://www.cerner.com/. Accessed 10 Aug 2020.

30. Hennink MM, Kaiser BN, Marconi VC. Code saturation versus meaning saturation:how many interviews are enough? Qual Health Res. 2017;27(4): $591-608$.

31. Hsieh H-F, Shannon SE. Three approaches to qualitative content analysis. Qual Health Res. 2005;15(9):1277-88.

32. Waring JJ. Constructing and re-constructing narratives of patient safety. Soc Sci Med. 2009;69(12):1722-31.

33. ledema R, Flabouris A, Grant S, Jorm C. Narrativizing errors of care: critical incident reporting in clinical practice. Soc Sci Med. 2006;62(1):134-44.

34. Gale NK, Heath G, Cameron E, Rashid S, Redwood S. Using the framework method for the analysis of qualitative data in multi-disciplinary health research. BMC Med Res Methodol. 2013;13:117.

35. Salkind N. Triangulation. In: Encyclopedia of research design. Thousand Oaks: SAGE Publications; 2012.

36. Nowell LS, Norris JM, White DE, Moules NJ. Thematic analysis: striving to meet the trustworthiness criteria. Int J Qual Methods. 2017;16(1): 1609406917733847

37. Lichtner V, Baysari M, Gates P, Dalla-Pozza L, Westbrook Jl. Medication safety incidents in paediatric oncology after electronic medication management system implementation. Eur J Cancer Care. 2019;28(6):e13152.

38. Endsley MR. Situation awareness in aviation systems. Handbook of aviation human factors; 1999. p. 257-76.

39. Daniel R, Schuck NW, Niv Y. How to divide and conquer the world, one step at a time. Proc Natl Acad Sci. 2015:112(10):2929-30.

40. Timmermans S, Berg M. Standardization in action: achieving local universality through medical protocols. Soc Stud Sci. 1997;27(2):273-305.

41. Reinhardt H, Otte P, Eggleton AG, Ruch M, Wohrl S, Ajayi S, et al. Avoiding chemotherapy prescribing errors: analysis and innovative strategies. Cancer. 2019;125(9):1547-57.

42. Mol A. The body multiple: ontology in medical practice: Duke University press; 2002.

43. Westbrook Jl, Ling L, Elin C, Lehnbom M, Baysari T, Braithwaite J, et al. What are incident reports telling us? A comparative study at two Australian hospitals of medication errors identified at audit, detected by staff and reported to an incident system. Int J Q Health Care. 2015;27(1):1-9.

\section{Publisher's Note}

Springer Nature remains neutral with regard to jurisdictional claims in published maps and institutional affiliations.

Ready to submit your research? Choose BMC and benefit from

- fast, convenient online submission

- thorough peer review by experienced researchers in your field

- rapid publication on acceptance

- support for research data, including large and complex data types

- gold Open Access which fosters wider collaboration and increased citations

- maximum visibility for your research: over $100 \mathrm{M}$ website views per year

At $\mathrm{BMC}$, research is always in progress.

Learn more biomedcentral.com/submission 